\title{
Entretien avec Dominique Schnapper
}

\section{Giovanni Busino}

\section{OpenEdition \\ Journals}

Édition électronique

URL : http://journals.openedition.org/ress/271

DOI : 10.4000/ress. 271

ISSN : 1663-4446

\section{Éditeur}

Librairie Droz

\section{Édition imprimée}

Date de publication : 1 août 2006

Pagination : 173-194

ISBN : 9-782-600-01108-2

ISSN : 0048-8046

Référence électronique

Giovanni Busino, "Entretien avec Dominique Schnapper », Revue européenne des sciences sociales [En ligne], XLIV-135 | 2006, mis en ligne le 13 octobre 2009, consulté le 04 mai 2019. URL : http:// journals.openedition.org/ress/271; DOI : 10.4000/ress.271 


\title{
Giovanni BUSINO
}

\section{ENTRETIEN AVEC DOMINIQUE SCHNAPPER}

\begin{abstract}
Pourquoi et comment avez vous choisi, ou accepté, d'être sociologue? Vous êtes plutôt réticente à parler de vous-même, avare de détails biographiques sur vos origines et sur votre formation intellectuelle, alors même que votre parcours pourrait donner un éclairage supplémentaire à celui de notre génération et faire mieux comprendre les raisons, les conflits, les déboires et les succès de la sociologie française d' aujourd' hui?
\end{abstract}

C'est vrai que je n'aime pas parler de mes états d'âme. Jusqu'à une date récente en tout cas, j'étais orientée vers l'avenir, je ne me penchais pas volontiers sur le passé. Plusieurs raisons sans doute. La première, c'est mon tempérament. Ma vie privée est privée. Je préfère écouter les autres raconter l'histoire de leur vie plutôt que de raconter la mienne. J'ai sans doute été renforcée dans cette attitude par Antoine, si discret, profond et contrôlé dans l'expression de ses sentiments.

Cela dit, ma réticence a été renforcée par ma filiation. Parler de moi, de mes origines ou de ma formation intellectuelle, c'était inévitablement parler de mon père. Il n'est jamais aisé de parler de son père, tant il fait intimement partie de soi. Dans ce cas, c'était particulièrement difficile, étant donné la position originale dans le «champ intellectuel», pour parler comme les sociologues, qu'occupait Raymond Aron à l'époque. Figure admirée et radicalement contestée, à la fois marginale et, à l'époque de mon entrée dans la vie professionnelles, centrale dans le monde de la sociologie puisqu'il avait la chaire de la Sorbonne. De plus, j'étais très sensible aux malheurs de la célébrité, aux malentendus qui naissent autour des personnalités connues, aux interprétations absurdes qu'elles suscitent. Elles étaient d'autant plus virulentes à l'égard d'un anticommuniste, qualifié d'homme de droite, que cette position, encore dans les années 1960, apparaissait peu élégante. Aron était isolé dans le monde intellectuel et même, plus largement, chez tous ceux qui se voulaient «dans le vent». La manière cavalière dont les étudiants de sciences-po parlaient de lui m'avait été particulièrement pénible. Je voulais préserver ma relation avec lui - et, plus tard, avec mon époux - de la moindre publicité. Mon père était, malgré les apparences, fragile et j'aurais, plus que tout, craint que la moindre déclaration pût être utilisée contre lui. C'est la raison pour laquelle j'ai toujours refusé de prendre parti dans la vie politico-médiatique. Je ne voulais pas apparaître comme un suiveur aveugle de ses positions, mais je voulais encore moins le critiquer ou même apporter la moindre nuance à ses analyses, ce

* Professeur honoraire à l'Université de Lausanne. 
qui aurait pu nourrir une attaque du style: «Même sa fille pense que..». Je crois que cela aurait été insupportable pour lui, et pour rien au monde, je n'aurais voulu le blesser.

Comment en être venue à la sociologie? J'avais eu en classe de philosophie (ce qu'on appelle aujourd'hui la Terminale) le coup de foudre pour la philosophie, j'avais donc l'intention de faire des études de philosophie. Mon père était réticent, il n'aimait guère le discours sous-heideggerien qui régnait alors dans les khâgnes, il le trouvait peu formateur. Mais j'ai gardé ce projet jusqu'à une expérience qui a été décisive. Mon ancien professeur de classe de philosophie, que j'avais beaucoup aimée, organisait chez elle des cours avec ses anciennes élèves où je me rendais avec enthousiasme. Mais, au cours d'une de ces séances, deux heures ont été consacrées à produire des affirmations dans lesquelles les phrases étaient déclarées également profondes et pleines de sens si on les formulait sous forme positive ou sous forme négative, alors qu'elles me semblaient également dépourvues de sens dans les deux cas. Tout cela avec des airs profonds. Je revois encore le salon où nous étions assises, certaines par terre, autour de notre «maître » et la conviction de mes camarades grisées de mots. Tout d'un coup, cela m'a paru totalement étranger. J'en ai conclu que, décidément, cette philosophie-là était un pur verbalisme, qu'elle ne m'apprendrait rien sur le monde, qu'elle n'était pas un véritable projet intellectuel. J'ai donc voulu répondre autrement à mes interrogations philosophiques. Il ne s'agissait pas de renoncer à la philosophie, mais d'essayer de partir de la connaissance de la réalité sociale pour répondre à des interrogations philosophiques.

Pratiquement, j'ai décidé, comme mon père le souhaitait, de quitter la khâgne pour entrer à Sciences-po. J'ai maintenant un jugement ambigu sur cette expérience. Du point de vue intellectuel, j’ai été déçue. Les enseignants, comparés aux philosophes et aux professeurs de khâgne que j'avais connus dans une grande khâgne parisienne, m'ont paru fort médiocres. Je me souvenais avec nostalgie de mon enthousiasme philosophique en terminale d'abord, puis en hypokhâgne, où Mme Dina Dreyfus donnait un enseignement de qualité exceptionnelle. De plus, j'avais aussi suivi à la Sorbonne les cours d'Henri Birault, philosophe talentueux et séducteur, dont les «petits cours» en dehors de l'université nous ravissaient. Les historiens et économistes de sciences-po donnaient un enseignement utile, mais ils me paraissaient dépourvus de toute ambition intellectuelle. La réalité se révélait bien prosaïque. A dix-huit ans, on est plus exigeant et on cherche des raisons d'enthousiasme. La recherche en science politique était encore peu développée et l'enseignement très professionnel: il s'agissait de fabriquer de futurs élèves de l'Ecole Nationale d'Administration. En revanche, ces années passées à l'Institut m'ont donné une certaine expérience du monde social. Je suis sortie - au moins intellectuellement - du monde dans lequel j’avais été élevée. Ni mon père ni nos proches ne pensaient en termes de carrière, ils pensaient en terme de vérité. La fréquentation de ces jeunes gens - les filles étaient encore peu nombreuses - pleins d'ambition sociale et sans véritable goût pour la chose intellectuelle - ils lisaient les best-sellers et fréquentaient les théâtres à la mode, ce qui me paraissait surprenant - m'a appris plus que beaucoup d'enquêtes sur la réalité sociale. Mais en même temps je ne m'y sentais pas bien, je n'ai gardé de ces années rue SaintGuillaume qu'une seule véritable amitié et fort peu de relations. En tout cas, j'ai été convaincue que j'étais faite pour une carrière intellectuelle - ce qui m'a enlevé 
toute tentation de présenter le concours d'entrée à l'ENA. Je ne pensais pas alors directement à la sociologie, d'autant qu'il n'existait pas de cursus universitaire de sociologie, mais j'avais acquis certains savoirs qui pouvait contribuer à la formation d'une sociologue.

Je n'avais, à la sortie de sciences po, aucun projet immédiat de «conversion» à la sociologie, sans doute à cause de la présence de mon père à la Sorbonne, dans un système universitaire centralisé où la Sorbonne jouait encore un rôle crucial. En l'absence de cursus universitaire de sociologie, l'entrée dans la recherche se faisait alors, après une licence de philosophie, d'histoire ou d'une autre formation (j'avais passé une licence d'histoire en même temps que je suivais les trois années de sciences-po), en participant aux enquêtes des «patrons » de l'époque, Crozier, Touraine, Mendras, Morin et quelques autres chercheurs du Centre de Recherches Sociologiques. On entrait comme «petite main» à l'occasion d'une enquête et, ensuite, si on avait fait ses preuves, le patron vous présentait au CNRS où l'entrée était facile. Un de mes amis, écrivain sans succès, s'était retrouvé au bout de deux ans d'une stratégie habile pilotée par Edgar Morin, orfèvre en la matière, chercheur en sociologie de la littérature au CNRS. Or, il m'était impossible d'entrer chez l'un ou l'autre de ces patrons qui entretenaient tous des relations ambiguës avec mon père et qui n'auraient évidemment pas souhaité se compliquer la vie en engageant sa fille. Ils n'avaient que l'embarras du choix pour recruter des «petites mains », la sociologie attirait les jeunes esprits et la vie de chercheur en séduisait beaucoup. J'ajoute que, de mon côté, je n'aurais guère été capable de faire ma cour au «patron», je suis farouchement indépendante et je sais mal me «vendre», sans doute par un mélange d'orgueil et de maladresse.

C'est à ce moment-là que j'ai rencontré Pierre Bourdieu, qui venait d'être nommé assistant de sociologie à la Sorbonne par mon père. Il nous paraissait aussi doué que loyal et généreux. Séducteur et brillant, il était plein d'ambition intellectuelle et voulait créer un centre de recherche sous la direction officielle de mon père - «pour lui », disait-il. Il m'a proposé de faire partie du projet. J'avais pour lui beaucoup d'admiration. Agrégé de philosophie, il comprenait mon intention profonde, il m'encourageait. Je croyais à son amitié. Nous avons tous besoin d'avoir un intercesseur pour entrer dans le monde intellectuel. Ce ne peut être votre père. Donc, pour moi, ce fut Bourdieu. Malgré toutes les désillusions, malgré le mal que je lui ai vu faire autour de lui, malgré le jugement moral que je porte aujourd'hui sur lui, je lui garde de la reconnaissance pour ce que j'ai appris grâce à lui. Il va de soi que son attention pour moi n'était pas étrangère au fait que, dans les premières années au moins, il avait besoin de mon père pour créer son propre centre de recherche - pour le dire en termes modérés. Je pense qu'il a aussi eu à un moment donné une amitié sincère pour moi, pour autant que l'amitié avait un véritable sens pour lui. Malgré tout, sans illusions, j'ai toujours gardé avec lui le lien qui lie un intellectuel à son introducteur au monde intellectuel.

Après des années difficiles à l'intérieur du Centre de sociologie européenne, mais qui étaient aussi des années fructueuses intellectuellement, nous nous sommes séparés après 1968. A partir de là, étant donné ce qu'était le monde universitaire après les «événements », j'ai mené ma petite barque sociologique seule. L'EHESS a été pour moi admirable et je n'ai jamais songé à la quitter, même quand l'hostilité que me manifestait Pierre Bourdieu, dont j'ai eu de nombreux témoignages, m'empêchait de progresser normalement. Personne ne m'a jamais 
reproché de ne pas jouer le jeu des échanges de services nationaux ou internationaux, des intrigues pour les élections, des responsabilités administratives qui donnent du pouvoir universitaire. Mieux, j'ai connu toute la reconnaissance modeste que peut accorder l'institution académique, puisque, une fois élue directeur d'études en 1981 grâce à François Furet, les présidents de l'Ecole m'ont toujours promue aussi rapidement que les règlements le permettaient. J'ai donc terminé ma carrière au sommet de mon corps. Je serai toujours reconnaissante à l'Ecole - et à l'université en général - de faire leur place aussi libéralement à des individus qui jouent aussi peu le jeu de l'institution et de leur permettre de mener une vie indépendante et libre, de travail et de réflexion. C'est un privilège dont je m'émerveille encore.

Elle m'a aussi permis d'assurer un enseignement qui a été pour moi une grande source de joie. J'ai aimé former de jeunes esprits à la sociologie et à la rigueur. J'ai finalement dirigé plus d'une trentaine de thèse et une douzaine de ces anciens «thésards» font maintenant une carrière universitaire. Je n'avais aucun pouvoir, je n'étais pas à la mode, donc je n'ai pas attiré les jeunes gens les plus ambitieux et les plus organisés. J'ai eu en revanche des étudiants sérieux, et de grande qualité humaine - c'était sans doute lié à mon style personnel. J'ai le sentiment que j'ai effectivement apporté à certains d'entre eux le sens de la chose intellectuelle. Mon séminaire n'était pas un lieu de pouvoir et l'atmosphère détendue qui y régnait leur a permis de s'épanouir intellectuellement et de prendre de l'assurance - c'est du moins ce que certains m'ont dit. Je leur ai fait une totale confiance et les meilleurs d'entre eux veulent bien penser que le climat de stimulation qui régnait dans le séminaire a été déterminant pour leur développement intellectuel. J'adaptais le séminaire à leurs travaux et je les associais à ma réflexion personnelle. Ce fut une expérience très enrichissante. En tous cas, eux m'ont beaucoup apporté. En dehors même de l'amitié, ils m'ont contrainte à défendre mes points de vue qui les choquaient souvent, à présenter en termes simples, mais rigoureux des analyses qui s'écartaient du discours commun. Et puis, il y a eu la véritable amitié et la collaboration intellectuelle constante avec Serge Paugam, François-Xavier Schweyer ou Corinne Rostaing, et d'autres moins proches quotidiennement mais également amicaux, qui ont été pour moi très précieux. L'Ecole, une fois encore, a reconnu mon travail, puisqu'un comptage récent a révélé que, malgré mon absence totale de pouvoir universitaire, j'ai été le directeur d'étude qui a obtenu le plus grand nombre de bourses de thèse pour ses étudiants. Tout le monde en a été surpris. Aujourd'hui, je suis reconnaissante à l'Ecole de m'autoriser à continuer mon séminaire, bien que je n'ai plus d'étudiants personnels, mais, même dans ces conditions devenues moins agréables puisque les étudiants sont formés par d'autres, je continue à y prendre plaisir.

En dehors de votre intérêt pour la philosophie et certains savoirs positifs, votre formation s'est parfaite à une époque où «les maîtres penseurs» et les «grands paradigmes unifiants» (fonctionnalisme, marxisme, structuralisme) étaient dominants à Paris. Ils englobaient tous les savoirs par totalisation et concevaient le social comme une "chose». Comment avez-vous pu échapper aux approches holistes et déterministes? Avez-vous été influencée par les écrits de ce "professeur dans la tourmente» qu'a été Raymond Aron? Votre première expérience de recherche a été la grande enquête, avec Alain Darbel, sur les agents $d u$ 
système administratif français. Les raisons de ce choix? Qu' est-ce qu' elle représente dans votre parcours formatif et pour la suite de vos travaux?

Je n'ai aucun goût pour les explications totales et pour les grands «paradigmes unifiants ». C'est très profond chez moi. L'idée de la multiplicité des facteurs, de l'ambiguïté du réel, des nuances qu'il faut apporter aux systèmes d'interprétation tient sans doute à mon tempérament, peut-être à ma formation d'historienne que je partageais avec Antoine. Même sans l'influence qu'exerçait sûrement mon père sur moi, bien que je n'en ai pas eu une conscience claire, je crois que j'aurais résisté aux grands «ismes», qui dominaient Paris. J'ai un réflexe de résistance à la mode, je n'aime pas penser comme tout le monde, j'ai mauvaise tête en somme.. D'autre part, j'avais renoncé à la philosophie justement pour me confronter à la réalité, pour élaborer une connaissance à partir des choses et non des mots, non pour adhérer à une pseudo-philosophie. Dans les cinq années de collaboration avec Bourdieu, j'ai cru partager cette attitude avec lui. A l'époque, il n'avait pas encore élaboré son «système » et prônait la connaissance empirique inspirée par la réflexion philosophique afin de dépasser l'opposition entre la sociologie scolastique enseignée par Gurvitch à la Sorbonne et les recherches empiriques directement influencées par la sociologie américaine, sans ambition théorique selon lui, qui se développait au Centre d'études sociologiques. Cette position me satisfaisait pleinement. Il m'apparaît clairement maintenant que, même sans les événements de 1968, j'aurais quitté Pierre Bourdieu un peu plus tard, car je n'aurais pas supporté de mettre en musique le «catéchisme» qu'il a progressivement imposé à tous les chercheurs de son Centre.

L'apprentissage de la sociologie s'est fait en deux étapes. D'abord, après avoir fait la connaissance de Bourdieu, je suis partie deux ans à Bologne où Antoine avait été nommé lecteur. Pour occuper mes loisirs, j'ai entrepris une thèse consacrée à la société bolonaise. Bourdieu me conseillait de loin mais très efficacement et j'ai fait un «terrain» dont je me souviens avec un immense plaisir, en découvrant progressivement les problèmes de la sociologie. Je vous rappelle que je n'avais pas fait d'études sociologiques spécialisées. Puis, à mon retour, j'ai été employée grâce à un contrat de recherche à la réalisation de l'enquête sur le public des musées qui s'est déroulée au Centre de sociologie européenne sous la direction de Bourdieu, pendant deux ans, entre 1963 et 1965. C'était la première grande enquête empirique à laquelle je participais et j'ai eu la responsabilité de son déroulement. J'ai évidemment beaucoup appris et je m'en souviens également avec bonheur. Mon apprentissage a été fait par la pratique. Je suis venue à la lecture des classiques et aux interrogations philosophiques à partir de cette pratique. Cela me convenait. Finalement, je n'ai pas été associée à la signature du livre qui est issu de l'enquête, L'Amour de l'art. Bourdieu m'a assuré que c'était un oubli de l'éditeur et j'ai voulu le croire, d'autant que j'étais pleinement consciente de tout ce que j'avais appris au cours de ces deux années. Certains de mes proches ont été convaincus que l'«oubli» était volontaire. Mon nom a d'ailleurs été rajouté à la deuxième édition, mais je n'ai jamais mentionné l'ouvrage dans mon curriculum vitae.

C'est pourquoi l'enquête sur les fonctionnaires n'est pas vraiment ma première enquête, mais la première que j'ai pleinement assumée avec Alain Darbel. C'était plus le fruit des circonstances que d'une vocation. Alain Darbel avait 
obtenu du Directeur de budget - probablement le fonctionnaire français le plus puissant à l'époque - un gros crédit pour une enquête sur la haute fonction publique. Parce qu'il ne pouvait l'assumer seul - il était en même temps administrateur de l'INSEE et plus statisticien que sociologue - et que Bourdieu souhaitait la récupérer dans le giron du Centre, parce qu'aussi, de mon côté, je souhaitais prendre un peu d'indépendance vis-à-vis du «patron », j'ai accepté, à sa demande, de m'en charger. Comme on s'intéresse à tout dans sa jeunesse et que mon projet intellectuel n'était pas encore très fixé, je m'y suis beaucoup investie et j'en garde un bon souvenir. J'ai fréquenté à cette occasion le milieu issu de sciences-po, me confirmant dans le bon choix que j'avais fait pour une carrière universitaire. Mais c'est au cours du déroulement de l'enquête que les événements de 1968 sont intervenus ainsi que la rupture avec Bourdieu qui s'en est suivie. Alain Darbel et moi avons donc terminé l'enquête sans qu'il intervienne - sinon pour la publier dans la collection du Centre de sociologie européenne chez Mouton. Ce travail a eu peu d'écho. Bourdieu ne l'a jamais mentionné. Le «terrain» de l'administration, à tous les niveaux, était solidement pris en main par Michel Crozier et son équipe. Alain Darbel est mort prématurément, peu de temps après la publication et je souhaitais changer de sujet. Nous n'avons donc pas exploité comme nous aurions pu le faire ce gros travail et, parfois, je pense que c'est dommage. Il arrive que les spécialistes de l'administration redécouvrent des faits ou des analyses que nous avions développées. Pour être reconnu dans un domaine, il ne suffit pas de publier un livre, même en deux volumes, il faut faire un véritable service après vente, multiplier les interventions et les publications annexes ou répétitives, se faire reconnaître comme «spécialiste». Etant donné les circonstances, nous n'avons rien fait de tout cela, et le livre a été quasiment oublié. En ce qui me concerne, cela a été formateur, j'avais appris beaucoup de la collaboration étroite avec un statisticien aussi compétent que sympathique et cela a élargi mon expérience sociale.

C'est pourtant à la fin de ce travail, en 1971, que je suis venue à des sujets qui m'étaient plus personnels et plus intimes. D'abord en rédigeant ma thèse sur Bologne puis en la prolongeant par des travaux sur les travailleurs migrants italiens.

\footnotetext{
Revenons si vous voulez bien sur deux moments de votre apprentissage sociologique.

Tout d'abord: comment avez-vous vécu les événements de mai 1968, perçu et jugé leur configuration intellectuelle? Ensuite: Après avoir soutenu la thèse sur "Les cent familles de Bologne», vous avez publié, en 1971, "L'Italie rouge et noire. Les modèles culturels de la vie quotidienne à Bologne», et puis, en 1974, une "Sociologie de l'Italie». Ces deux livres, reçus avec suspicion par les sociologues alors à la mode, marquent pourtant la singularité et la nouveauté d'une démarche dans un domaine contrôlé par les marxistes, l'empirisme et par ce que Sorokin appelait la «quantophrénie».

Dans ces deux livres la dimension anthropologique semble évidente. Mais peut-on dire que c'est d'elle que vous faites dépendre la forme politique que se donne la société?
}

Vous me forcez à me poser des questions que j'ai toujours eu du mal à aborder de front, le lien entre mes recherches des années 1970, disons jusqu'au milieu des 
années 1980 et les travaux qui, à partir de la fin des années 1980, ont abouti à mes livres, La France de l' intégration et la suite, ce qui représente le véritable tournant dans mon itinéraire. Je vais essayer de le faire.

Je reviens d'abord à votre première question sur les événements de 1968. Ils ont été pour moi extrêmement pénibles. Ils ont fait éclater le Centre de sociologie européenne en révélant au grand jour une opposition intellectuelle larvée, mais profonde, qui existait entre Bourdieu et mon père. Les membres du Centre, qui n'étaient pas des amis mais que je croyais être des camarades loyaux, se sont joints à Bourdieu, qui était leur véritable patron, d'une manière qui m'a paru absurde, adoptant le vocabulaire pseudo-révolutionnaire pour mener une lutte contre la «tyrannie» du mandarin, mon père, qui ne s'occupait guère de la vie quotidienne du Centre et n'a jamais tyrannisé personne, il avait même de la peine à assurer son autorité sur sa secrétaire personnelle. Bien entendu, il y avait bien autre chose que ce qui était dit: l'hostilité politique à l'égard d'Aron, le besoin de s'affirmer seuls avec Bourdieu, en évitant le patronage, même lointain et bienveillant, du «vieux» directeur. Mais ces discours me paraissaient absurdes. J'ajoute que j'étais enceinte et peut-être particulièrement sensible aux agressions verbales. Je dis bien verbales, parce qu'il n'y a jamais eu d'agressions autres que verbales et qu'à aucun moment je n'ai eu peur. Mais évidemment ma situation au Centre, entre Aron et Bourdieu, était la moins confortable. J'avais peine à écouter ce langage révolutionnaire, alors que nous étions tous des petits bourgeois plus ou moins nantis, mais installés dans le confort de la fonction publique pour le reste de notre vie, nourris par la République et laissés parfaitement libre de la critiquer. J'avais un mélange de dégoût et d'indignation. Je me sentais très étrangère au mouvement dans la forme qu'il prenait, en tous cas dans les laboratoires de sciences sociales. Je me souviens d'un épisode comique. Au milieu de la «révolution», Edgar Morin a demandé un rendez vous à mon père. J'étais un peu inquiète, car Morin était au coeur du mouvement. Or, mon inquiétude était bien vaine, il était venu pour l'entretenir de ses problèmes de carrière au CNRS ! C'était légèrement surréaliste pour un «révolutionnaire». Cela dit, il me semble me souvenir qu'en dehors de l'expérience personnelle que nous en faisions dans les centres de recherche en sciences humaines, j'essayais de comprendre la révolution «démocratique» que symbolisaient les événements de 1968. Je me souviens précisément d'avoir évoqué avec mon père l'épuisement des formes d'autorité traditionnelle, l'Eglise (il y avait eu révolte chez les jésuites..), l'Etat, les partis politiques (les gauchistes contre le parti communiste), la famille, la libéralisation des mœurs, l'université. Rétrospectivement, je suis sensible au fait que c'était l'entrée - dans le style révolutionnaire ou pseudo-révolutionnaire propre à la tradition française qui ne se remet pas de la Grande Révolution - dans une étape nouvelle de la démocratie, dans laquelle, comme dit Tocqueville, tout est devenu permis. Je n'arrivais pas à condamner cette évolution comme le faisaient les conservateurs, je la considérais plutôt en sociologue, mais je m'indignais, à cause de ma conception même de la sociologie, que mes collègues sociologues participent, au premier degré, à ce mouvement, comme si leur pratique professionnelle ne servait qu'à donner des arguments à la «révolution» au lieu de la relativiser, de l'apprécier, de la critiquer et de la comprendre. Or, certains d'entre eux n'hésitaient pas à affirmer que leur activité de chercheurs les faisait participer à la révolution en cours, qu'ils luttaient avec les «masses » et les «classes défavorisées » 
contre «les dominants». Quand ils avaient des soucis de carrière, ils expliquaient que c'était l'effet de la tyrannie du gouvernement, alors qu'à l'époque on faisait carrière en étant gauchiste! Bref, il me semblait qu'il y avait là une mauvaise foi assez insupportable. Cette position n'a pas favorisé ma carrière, d'autant que mon père était devenu le symbole de la réaction. Naturellement j'ai quitté le Centre de Bourdieu et j'ai été rattachée au centre de Recherches historiques de l'Ecole des Hautes Etudes, où je suis restée jusqu'aujourd'hui. C'est un rattachement surtout administratif, mais les directeurs ont toujours été très bienveillants à mon égard et il convenait à ma marginalité.

Cette marginalité explique que mes travaux anthropologiques des années 1970-1985 soient restés sans écho. Comme vous le dites, je n’étais pas «dans le vent », n'étant ni marxiste ni quantitativiste. Bien entendu, je reconnais pleinement, cela va de soi, la nécessité et la valeur des recherches quantitatives lorsqu'il s'agit de répondre à un certain nombre d'interrogations globales sur l'évolution des sociétés, comme la répartition de la fortune ou les inégalités. C'est par les données statistiques qu'on peut apprécier la valeur de la théorie marxiste de la paupérisation relative ou absolue. Mais c'est vrai que j'ai une préférence pour les méthodes dites qualitatives. Est-ce parce que j'ai été marquée par ma première enquête à Bologne? Est-ce ma formation d'historienne? Mon admiration pour les premiers travaux, anthropologiques, de Bourdieu en Algérie et au Béarn? Est-ce une question de tempérament, le goût du concret et des relations directes avec les populations étudiées? Je ne sais pas. Toujours est-il qu'il me semble qu'une enquête de type anthropologique, à condition de garder à l'esprit la critique inhérente à toute enquête sociologique, est fructueuse et, finalement, répond à la plupart des questions que le sociologue se pose. En ce qui me concerne, en dehors de l'enquête sur l'administration, la formalisation statistique a confirmé ou affiné les résultats de mes recherches, elle ne m'a pas permis de les « découvrir ». Il va de soi que la confirmation statistique est nécessaire, mais elle me paraît moins excitante intellectuellement que la réflexion et l'expérience directe. Par exemple, dans La France de l'intégration, sur les processus d'intégration des populations migrantes, j'ai mobilisé la connaissance historique, les nombreuses études partielles des spécialistes, souvent des thèses de doctorat, mes quelques enquêtes personnelles, également partielles, pour rédiger les deux chapitres concernant les migrants et leurs descendants. Alors que le livre était déjà publié, l'INED et l'INSEE se sont associés pour mener, à l'échelle nationale, une enquête sur l'intégration des descendants de migrants. Si l'on laisse de côté les méthodes et les chiffres et qu'on se penche sur les seules conclusions sociologiques de l'enquête, il n'y a aucune véritable «découverte», sinon que le sort des jeunes filles maghrébines est moins favorable que l'on pensait à partir des enquêtes partielles. Bien entendu, cela ne remet aucunement en question la légitimité de l'enquête nationale. Les résultats des recherches n'ont pas le même statut, n'ont pas le même sens selon la manière dont ils sont obtenus et une enquête représentative au niveau national est essentielle pour faire avancer la connaissance. Elle apporte, malgré les critiques qu'on a pu lui faire, la légitimité statistique aux analyses sociologiques. Reste que la réflexion de type historique et anthropologique ne sort pas disqualifiée de la confrontation. François Héran, directeur de l'INED, l'a d'ailleurs remarqué avec une grande élégance. Encore une fois, il ne s'agit pas de déclarer une méthode supérieure à l'autre, mais seulement de 
souligner que la méthode anthropologique, que j'ai pratiquée sans doute par goût personnel, peut être, sous certaines conditions, fructueuse. La première condition est évidemment de se poser des questions proprement sociologiques - et non de se contenter de la description comme le font certains anthropologues du présent - et de garder l'attitude critique de la sociologie que l'enquête statistique formalise. Ainsi il faut se demander sans cesse si les résultats de l'enquête sont dus à des circonstances particulières ou peuvent être généralisés, il faut les relativiser et se demander, chaque fois qu'on interprète un résultat, s'il ne faut pas faire intervenir un autre facteur. Dans les termes de la sociologie quantitative, il faut faire intervenir les variables-tests dans le raisonnement, etc. La logique d'interprétation des résultats n'est pas différente lorsqu'il s'agit de résultats obtenus par questionnaires ou par une enquête plus anthropologique.

Plus généralement, ce n'est pas seulement une question de méthode, cette préférence anthropologique implique une certaine conception du social, l'idée que le collectif est présent même dans un milieu peu nombreux et qu'aucune enquête ne peut être interprétée en dehors d'une réflexion historique et même philosophique. Il est vrai que cette position m'a longtemps marginalisée.

J'en viens à votre dernière question, le lien entre cette «dimension anthropologique » et la forme politique, en d'autres termes, sur le lien entre les deux étapes de mes travaux. Pendant une dizaine d'années, j'ai pensé que le concept qui organisait mes travaux portant sur les migrants et leurs descendants, les identités juives ou l'épreuve du chômage était celui d'identité: pour toutes ces populations, j'analysais une crise identitaire - par exemple, dans le cas du chômage - ou, en tous cas, d'une interrogation sur l'identité - qu'est-ce qu'être juif? Le concept me renvoyait à l'idée, somme toute banale, que, dans les sociétés démocratiques, les identités n'étant pas données par la naissance, les individus s'interrogeaient sur leur propre identité et sur leur statut et qu'on pouvait comprendre en profondeur nos sociétés à partir de cette interrogation. Dans la perspective anthropologique qui était alors la mienne, j'avais le sentiment que ce concept donnait un cadre à des travaux divers par les populations concernées, mais où se déployaient une même perspective et une même méthode. J'avais donné à ma direction d'étude le nom d'《ethnosociologie des sociétés modernes », voulant montrer par là que je m’efforçais de répondre par les méthodes généralement utilisées par les ethnologues ou les anthropologues aux questions plus spécifiques de la sociologie. J'ai découvert progressivement la dimension politique au fur et à mesure du développement de mon travail. Ma participation à la Commission de la nationalité a été un instrument de cette évolution puisqu'elle m'a conduite à poser en termes proprement politiques mes travaux sur les identités particulières. La France de l'intégration en est directement issu.

La mise en évidence des modèles culturels et des relations sociales les plus quotidiennes (ainsi que vous les décrivez dans vos travaux sur l'Italie) me semblent ouvrir la voie à une autre manière d' aborder la problématique du lien social et la question de l'identité collective. Lorsque je les ai relus il y a quelque temps, j'y ai entrevu un lien très fort avec l'étude sur les émigrés italiens en France et aux Etats-Unis et surtout avec l'enquête synthétisée dans le volume "Juifs et Israélites». Dans la préface à ce dernier volume vous portez un regard inéquitable sur vos premiers travaux. Pouvez-vous nous en dire davantage? En allant 
plus loin que les modèles culturels des ethnologues et la permanence du passé des historiens, où avez-vous voulu enraciner le lien social? Comment caractérisezvous les relations entre le lien social et l'identité individuelle et collective?

C'est vrai que l'ensemble de mes travaux, depuis Bologne jusqu'à l'enquête sur les identités juives - près de vingt ans finalement -, ont une inspiration commune. Ils reposent sur les mêmes méthodes que j'ai essayé d'expliciter dans la préface de Juifs et israélites, proches de celles que mobilisent les anthropologues: observations prolongées, entretiens approfondis et échanges personnels. Elles me paraissaient plus adaptées à des interrogations qui portaient toutes - je m'en rends compte rétrospectivement - sur le sens que les individus donnent à leur existence. A travers ce qui était sans doute perçu comme une position «culturaliste», donc peu appréciée dans le monde sociologique, c'était en fait - à travers ces enquêtes minutieuses sur les conduites et sur les discours grâce auxquels les individus donnent un sens à leur existence - une interrogation sur les identités et sur le lien social. Tous les sociologues s'interrogent sur le lien social, presque à titre professionnel. Mais je formulais à mon tour cette interrogation fondatrice du projet sociologique par le biais du rapport au temps quotidien (vie quotidienne à Bologne, pratiques juives parfois présentes quotidiennement ou expérience de l'ennui quotidien lié au chômage), de l'affirmation identitaire (qu'est-ce qu'être juif, qu'est-ce qu'être un immigré italien?) ou de la crise de l'identité qui survient lors de l'épreuve de l'immigration ou du chômage. Or, l'on sait que les identités se définissent et se renouvellent dans la relation à autrui, ce qu'on peut baptiser le «lien social». J'avais donc le sentiment que ces enquêtes me permettaient de toucher à quelque chose d'essentiel: comment les hommes renouvellent chaque jour le sens de leur destin social. Seule l'enquête de type anthropologique permettait cette démarche.

En même temps, les résultats de ces enquêtes ne prenaient sens que dans une histoire. Comment comprendre la société bolonaise des années 1960 sans se rappeler le rôle des «régions » en Italie et du patriotisme local, la longue domination de la papauté sur les Etats du Pape et, plus généralement, la présence de l'Eglise catholique, le passé fasciste et le rôle du parti communiste? Comment comprendre les identités juives des années 1970 en faisant abstraction de l'antisémitisme, du patriotisme des «israélites » d'avant la seconde guerre mondiale, de la shoah, de l'Etat d'Israël? Et on ne comprend rien non plus à l'épreuve du chômage si l'on fait abstraction de l'histoire de l'Occident qui fait de la production des richesses le centre de la vie collective. Inscrire les résultats de l'enquête anthropologique la plus minutieuse et la plus approfondie dans une histoire très large et très ancienne était essentiel. En même temps, j'entendais ne pas renoncer aux interrogations propres de la sociologie sur la société contemporaine. J'avais essayé de les formuler dans la conclusion de Juifs et israélites d'une manière que je trouve aujourd'hui pas très satisfaisante, trop scolaire et prudente. C'est ce que j'ai repris et développé dans la seconde étape de mon itinéraire.

En lisant «Juifs et Israélites» j' ai eu l' impression que pour vous les collectivités humaines se caractérisent par leur histoire plutôt que par des traits raciaux ou ethniques. Pourtant ici et là vous faites allusion à une identité de groupe, à une différence "groupale» en tant que distinction. Que faut-il entendre par «collectivité historique», par «identitéldifférence de groupe»? 
Y-a-t-il une dimension «politique» dans ce livre de 1980 traitant de façon si novatrice et originale d'un thème quasi éternel?

Quel écho a eu chez les sociologues français et chez les intellectuels occidentaux vos travaux parus avant les années 1980 ?

Il n'y avait aucune dimension politique dans le livre sur les identités juives, tel que je l'avais conçu à l'époque. Je restais dans une perspective très anthropologique. J'ai moi-même souligné les limites de ce livre dans La compréhension sociologique. L'enquête elle-même était née d'une remarque d'Annie Kriegel à la suite de la publication d'un de mes articles sur les immigrés italiens: «Vos Italiens deviennent français, mais les juifs, eux, restent juifs ». Je ne sais pas si c'est entièrement vrai, mais cette réflexion m'a frappée et décidée à entreprendre cette enquête que j'avais seulement très vaguement envisagée jusque là. Mais je restais dans la réflexion sur les identités sociales, c'était le prolongement direct des travaux concernant les immigrés italiens ou les chômeurs, sur les réinterprétations des identités sociales. Dans tous les cas, la dimension historique me paraissait essentielle, et c'est bien ce que j'ai essayé de traduire, quinze ans plus tard, par le concept de «collectivité historique», qui permet de dépasser les débats qui opposent les sociologues américains des relations interethniques sur l'emploi du concept de «groupe racial» et de «groupe ethnique», débats liés à la particularité de la situation américaine concernant les Afro-Américains. «Collectivité historique» est un concept pour moi essentiel, puisqu'il fonde l'identité collective sur une histoire partagée, à la fois réelle et imaginaire, et non sur des traits «raciaux» ou «ethniques». L'exemple des juifs est à cet égard idéal-typique, puisqu'il n'existe ni «race» juive ni même contenu «ethnique» d'une culture juive; ce qui relie les juifs objectivement si différents les uns des autres, c'est l'existence ou même simplement l'idée d'une histoire partagée. Jusqu'à présent, le concept n'a été utilisé que par mes étudiants, mais je ne désespère pas que sa fécondité soit un jour plus largement reconnue! Je ne me souviens pas d'avoir beaucoup utilisé «l'identité de groupe», sinon dans un sens assez vague.

Quant à l'écho de mes travaux, j'ai longtemps eu le sentiment qu'il était nul. Bourdieu m'avait fait dire par Isac Chiva que Juifs et Israélites, sur un sujet aussi difficile et idéologique, manifestait la maîtrise d'un véritable sociologue. Cela m'avait fait plaisir, naturellement. Chiva portait le même jugement. Mais le livre n'a donné lieu à aucune réunion ou discussion parmi les sociologues. Les livres de sociologie des juifs risquent toujours de tomber dans un no man's land. Les nonjuifs sont gênés par un sujet qu'ils connaissent mal et qu'ils savent sensible, quant aux juifs eux-mêmes, ils détestent cette objectivation et recherchent des ouvrages qui célèbrent leur culture, leur malheur ou, en tous cas, leur recherche d'identité. Malgré la traduction en anglais à Chicago University Press, grâce à Edward Shils, l'écho du livre est resté fort limité, mais je crois qu'il est reconnu comme une étape par les quelques sociologues sérieux qui s'intéressent à ces problèmes. Reste que je fréquentais si peu les milieux sociologiques que j'ai eu l'impression, jusque dans le milieu des années 1990, que mes recherches tombaient dans un grand vide. Elles n'ont jamais été discutées. Aussi j'ai découvert avec une grande surprise que L'épreuve du chômage, quelques années après sa parution, était devenu un classique de la formation des jeunes sociologues dans les universités. J'en ai effectivement vendu de l'ordre de 25000 exemplaires, ce qui est beaucoup 
pour un livre de sociologie. Mais cela s'est fait comme en dehors de moi, sans que les sociologues reconnus et les institutions en débattent. J'avais quelques amitiés dans le milieu, mais elles ne suffisaient pas pour que mes travaux soient véritablement pris en compte. Je garde encore de la reconnaissance à François Isambert qui m'a demandé dans les années 1980 de présenter mes travaux dans une série d'interventions présentant la sociologie française aux étudiants de l'EHESS. J'avais l'impression que c'était la première fois qu'un collègue sociologue prenait quelque intérêt à mes recherches. Entre les marxistes, les quantitativistes et la logique de pouvoir des différentes «écoles» qui se partageaient le champ de la sociologie, j'ai toujours eu le sentiment que ma place était inexistante. Il devait tout de même y avoir une forme de reconnaissance, puisque j'ai été élue directrice d'études à l'EHESS en 1981, mais peut-être était-ce surtout dû à l'influence du président, François Furet, sur l'assemblée des Hautes Etudes. J'ai d'ailleurs cru comprendre, à travers une réflexion discrète de Jacques Revel, qui était alors le collaborateur le plus proche de Furet, que cela n'avait pas été si facile.

Votre enquête "L'épreuve du chômage» a été publiée en 1981. Vous revenez sur cette même problématique dans un texte de 1996 paru dans le volume collectif intitulé «L' exclusion» et puis en 1997 dans votre "Contre la fin du travail». Vous écrivez que le chômage désocialise, fragilise la dignité personnelle, réduit les échanges sociaux et plonge le chômeur dans une grande solitude. Pour vous, c'est le rôle du travail de produire l' intégration sociale, de façonner et étendre les liens sociaux.

Si le chômage produit l' exclusion, si celle-ci affaiblit le sens d'appartenance au monde commun, comment obvier à ces inconvénients dans nos sociétés où le travail devient de plus en plus un bien rare et où la souveraineté individuelle et les défenses corporatives ne favorisent guère la réalisation des programmes collectifs de solidarité?

C'est en effet une des grandes inquiétudes qu'on peut avoir sur le destin de nos sociétés. L'affaiblissement des traditions et des institutions issues de la tradition a laissé un rôle central et presque unique au travail comme instrument d'intégration sociale - préparé par l'Ecole. C'est par le travail que se construit la dignité des hommes dans des sociétés organisées autour de la production des richesses. Or, nous assistons à une double dévalorisation du travail: par rapport au capital et par rapport à l'assistance. La domination d'un capitalisme de plus en plus financier remet en question la valeur accordée au travail. D'autre part, les exigences de la citoyenneté conduisent à développer un Etat -providence qui, de plus en plus, ne s'inscrit plus seulement dans la logique de la redistribution, mais dans celle de l'assistance. Les revenus liés à l'assistance s'accroissent (même s'ils sont insuffisants pour chaque individu) pour des raisons d'exigence démocratique fort compréhensibles et justifiés. Mais, du coup, le rôle de Grand Intégrateur qu'a joué le travail dans nos sociétés est remis en cause. Les travailleurs sont humiliés que leur travail soit moins bien rémunéré que le capital. Les assistés sont humiliés par le fait que l'intégration par l'assistance est une intégration au rabais, fondée sur l'inégalité, qui consacre l'incapacité et l'inutilité de celui qui en bénéficie. C'est là, je crois, à long terme, ce qui est le plus préoccupant pour l'intégration des sociétés démocratiques. 
Après les enquêtes empiriques sur les diverses formes de marginalité (les immigrés italiens, les juifs, les chômeurs), vous publiez «La France de l'intégration. Sociologie de la Nation en 1990» (1991), «L'Europe des immigrés» (1992) et «La communauté des citoyens. Sur l'idée moderne de Nation» (1994).

Peut-on dire que les débats publics très brûlants au sein de la Commission sur la nationalité vous ont obligée à regarder en philosophe politique et en historien vos propres recherches en sociologie empirique?

Certains ont qualifié ces livres d' essais politiques, d' autres d' illustration de la doctrine «souverainiste», alors que vous dites clairement de proposer une interprétation globale des processus d' intégration et élaborer une théorie de la Nation en tant que valeur et nécessité principales, à l'époque actuelle, du lien social. Pourquoi, d'après vous, une telle méprise interprétative?

Si la Nation est un projet politique universaliste basé sur la citoyenneté, de quelle façon étendre le lien social aux non citoyens et à ceux prônant des traditions identitaires communautaristes, des particularismes religieux et historiques?

Les débats à l'intérieur de la commission de la nationalité ont été pour moi l'occasion de prendre une distance à l'égard de mes propres travaux et de les objectiver. Cela a été aussi l'occasion d'apprendre du droit, ce que j'ai trouvé passionnant. J'avais jusque là sous-estimé le sens et l'effet des règles juridiques à cause d'une perspective restée très anthropologique. Les nombreuses séances de réflexion commune, de discussions passionnées et d'apprentissage grâce aux travaux de la Commission m'ont conduite à une réflexion à la fois plus politique et plus philosophique. La France de l'intégration, qui est un livre de synthèse, en est directement issu. C'est pourtant un ouvrage sociologique en ce sens que la réflexion est fondée sur les résultats de la connaissance objective de la société rassemblée par les sociologues. Pourquoi a-t-il été critiqué par certains sociologues comme n'étant qu'un «essai politique »? Laissons de côté l'hypothèse de quelque jalousie liée à ce que ces travaux ont été lus au-delà du public des sociologues universitaires. L'essentiel est que les sociologues ont singulièrement réduit leur ambition intellectuelle. Les fondateurs de la discipline se posaient des questions fondamentales et philosophiques sur l'évolution de nos sociétés. Aujourd'hui, le caractère académique de la recherche a conduit beaucoup de sociologues à rechercher des résultats peut-être de plus en plus précis, mais sur des sujets de moins en moins intéressants, pour reprendre une réflexion ironique de mon père. Il me semble pourtant que, sur les sujets qui sont à l'origine de l'interrogation sociologique, tels que l'intégration sociale, les formes renouvelées des liens sociaux ou l'identité nationale, les sociologues peuvent contribuer utilement au débat intellectuel. Ils ont leur place à côté des philosophes politiques, qui risquent toujours de devenir normatifs et de fonder leur réflexion sur la seule connaissance intuitive qu'ils ont de la société à partir de leur expérience sociale. Les sociologues, eux, ont le mérite de s'appuyer sur la connaissance objective de nos sociétés, obtenue par des méthodes rigoureuses, donc de relativiser leur propre expérience sociale. Ils s'efforcent de comprendre plutôt que de juger et de conseiller. Ils prennent en compte les manières concrètes dont s'appliquent les règles et les principes. Ils peuvent donc renouveler, à partir de cette connaissance, la réflexion de nature philosophique sur notre destin collectif dans les sociétés démocratiques modernes. En tous cas, c'est ce que j'ai tenté de faire. Je 
regrette que de nombreux sociologues limitent, de ce point de vue, leur projet et se contentent trop souvent, quand ils traitent des problèmes de nos sociétés, de se rallier à une dénonciation radicale, qui est confortable pour eux, mais n'aide pas beaucoup les non-sociologues à comprendre le monde dans lequel nous vivons.

Par ailleurs, si l'on m'a attribué une position «souverainiste» qui n'était pas la mienne, à la suite de La communauté des citoyens et de son succès public, c'est que mes livres sont parus lors d'un débat entre «républicains » et «multiculturalistes », dans lequel ma position était évidemment plus proche des premiers, mais qui ne correspondait pas à mon intention qui, encore une fois, était de faire avancer la réflexion fondée sur la connaissance objective, non de prendre des positions politiques - même si je n'ai pas la naïveté de penser que les deux soient totalement indépendants. Catégoriser les positions, fut-ce en les caricaturant, fait partie du fonctionnement du monde intellectuel parisien. Et il est probablement inévitable que, sur des sujets de ce genre, l'interprétation politico-médiatique soit dominante. La position sociologique n'est pas naturelle, elle est le produit d'un effort long et jamais totalement acquis. J'avais pourtant, me semble-t-il, clairement précisé mon projet, mais il aurait fallu lire le livre. Une anecdote à ce propos. Le Centre de sociologie des organisations m'avait invitée à discuter $L a$ communauté des citoyens avec les chercheurs du laboratoire. Le livre était présenté par l'un d'entre eux, Jean-Pierre Worms, réputé tenant du «multiculturalisme». La présentation et la discussion qui ont suivi ont été excellentes, la meilleure de toutes celles auxquelles j'ai participé. J'ai donc chaleureusement remercié Jean-Pierre Worms à la fin de la réunion. Il m'a répondu: «Je vais vous faire une confidence, quand le livre est sorti, je l'ai vu chez un de mes amis et je l'ai feuilleté quelques minutes, et je me suis dit, c'est de la philosophie républicaine, sans intérêt. Mais quand je l'ai lu de près, pour préparer cette séance, j'ai vu que cela était beaucoup plus intéressant!» Je l'ai remercié de sa franchise. Mais le premier épisode qu'il racontait était très typique de la vie intellectuelle parisienne: on catégorise avant de lire. Le second épisode intervient plus rarement, de toute évidence, et, en plus, les lecteurs ne sont pas tous de la qualité de J.-P. Worms! J'ajoute que j'ai souvent présenté mes travaux chez les non sociologues, mais que jamais on ne m'a invitée dans une instance comme celle qu'organise Gérard Mauger «Lire les sciences sociales». Mes travaux ont eu finalement plus d'écho en Suisse, en Belgique et surtout au Québec. Dans ce dernier cas, j'ai été particulièrement sensible au fait que les Québécois trouvent mes travaux utiles pour leur réflexion et pour leur action, car ils pensent ces problèmes d'identité nationale et civique «en situation».

Pour répondre à votre question sur le lien social, dans la société organisée par le principe, les valeurs et les pratiques de la citoyenneté - qui jusqu'à présent a toujours été nationale mais ne l'est pas nécessairement - il existe une tension, inévitable, entre les dimensions «ethniques » de la vie collective - les liens historiques et culturels qui unissent les hommes au niveau national, infra-national ou supra-national - et le principe civique de transcendance de ces particularismes par la citoyenneté commune. Quand le sentiment national et la foi dans les valeurs civiques sont suffisamment forts, ils priment l'identification aux particularismes religieux ou historiques qui, bien sûr, continuent à exister, mais sur un mode politiquement mineur. L'affaiblissement de la dimension civique et la dissolution du sentiment national et patriotique - historiquement les deux ont été liés - laisse sa 
place au développement des identifications particulières, qu'elles soient religieuses, régionales ou «ethniques». C'est ce à quoi on risque d'assister quand s'affaiblit le sentiment d'affiliation à une communauté politique et civique et que s'accroît le sentiment du «droit à»l'expression de toutes les formes de l' «authenticité » de chacun.

Dans «La relation à l'autre» (1998), vous nous donnez une admirable réflexion sur la citoyenneté, sur l' universalisme et le particularisme. Vous nous parlez également du relativisme culturel et des conditions sociales. Comment peut-on passer d'un groupe culturel «distinct», de la condition sociale "différente» à la citoyenneté basée sur des principes universels, ancrée dans cette sorte de religion civile qu'est le patriotisme républicain?

Dans «La compréhension sociologique» (1999), vous jugez la méthode typologique féconde car elle permet d'analyser les liens entre les conduites des individus. Est-ce que le passage du particularisme à l' universalisme s'effectue grâce à des séquences comportementales plausibles conférant le sens à la mise en relation de grandeurs agrégées?

Je vous remercie de votre appréciation de La relation à l'autre, car c'est pour moi, un livre essentiel. Comme il est apparu comme trop «savant»-y compris à des chercheurs du CNRS qui m'ont demandé si j'avais vraiment lu tous les livres dont je parlais! -, il n'a connu qu'une diffusion honnête, mais limitée à un public spécialisé et il n'a été traduit qu'en turc et en bulgare! Or, il résume le coeur de mon entreprise. Je vais essayer de répondre à votre interrogation, bien que je ne sois pas sûre de la comprendre complètement. Le caractère utopique de la citoyenneté tient précisément en ce qu'elle entend transcender la « distinction » des divers groupes culturels réunis dans la même entité politique et les «différences», donc, dans les sociétés démocratiques, les «inégalités» qui séparent les groupes sociaux. Cette transcendance ne peut être complète, bien entendu. Les groupes culturels continuent à exister et les inégalités sociales, également. Les tensions entre l'universalité de la citoyenneté et la réalité des différentes allégeances culturelles et des inégalités des conditions sociales sont constitutives de la société démocratique. Mais la transcendance républicaine à vocation universelle, ellemême, tend à se constituer effectivement en une «sorte de religion civile» susceptible de trahir sa vocation universelle. C'est inévitable, parce que la société n'est pas formée de citoyens, ou de sujets de droits, mais d'individus historiques, fidèles à certaines traditions historiques et connaissant une condition sociale particulière. Une société politique ne pourrait les intégrer et les mobiliser si elle invoquait seulement des principes abstrait comme la démocratie ou la citoyenneté. J'ai toujours été très frappée du fait que pendant la Seconde guerre mondiale, les enquêtes sociologiques ont montré que les combattants, loin d'être mus par le désir de défendre les grands principes de la démocratie contre l'hitlérisme, étaient mobilisés par le nationalisme et la solidarité dans le combat qui les liait à leurs camarades. Roosevelt n'aurait peut-être pas réussi à entraîner les Américains dans la guerre pour la démocratie sans l'assaut des Japonais sur Pearl Harbor, qui s'attaquait directement à l'honneur de la nation et vous connaissez les résultats des enquêtes classiques des sociologues sur les combattants de l'armée américaine. Ces résultats, mutatis mutandis, peuvent s'appliquer à la société démocratique. 
Elle est de fait intégrée par les institutions, au sens le plus concret du terme - jusqu'à présent nationales - et par les relations entre les individus qui donne sens et efficacité à ces institutions. Une nation n'est jamais purement civique, elle est indissolublement ethnique et civique - même si c'est l'ambition de la transcendance civique qui singularise les sociétés démocratiques.

Ce que j'ai essayé de théoriser - ou simplement de préciser - dans La compréhension sociologique, c'est une pratique qui permet d'analyser les conduites des hommes en conjuguant la perspective macrosociologique et les analyses microsociologiques que multiplient les sociologues. C'est en effet dans cet entredeux - dans le passage entre le macrosociologique et le microsociologique - que me paraît résider la spécificité du point de vue sociologique et sa fécondité. Certains philosophes politiques font des variations brillantes sur la démocratie, qui sont fondées sur leur expérience sociale personnelle en négligeant les résultats des enquêtes sociologiques. Résultat, leur réflexion porte sur les classes moyennes et intellectuelles qu'ils connaissent - d'autant que c'est aussi bien souvent le cas des médias. De leur côté, beaucoup de sociologues s'en tiennent à des analyses étroites en refusant de se poser les problèmes de la sociologie que se posaient les «fondateurs », qu'ils dénoncent désormais comme pré-scientifiques ou «philosophiques». Il me semble qu'ainsi ils négligent ce qui fonde la légitimité de leur point de vue. C'est en essayant de s'interroger sur les sociétés modernes à partir d'une connaissance précise - acquise grâce aux démarches statistique et anthropologique - que le sociologue justifie la spécificité de sa démarche. Les analyses typologiques sont un bon instrument de recherche dans ce projet, puisque la logique qui sous-tend la construction d'une typologie est liée aux caractéristiques macrosociologiques de la société démocratique. D’une certaine façon, on trouve tout dans les relations les plus individuelles, en particulier cette passion de l'égalité qui a redéfini les relations familiales comme les relations entre les intervenants de la «communauté éducative», comme l'on dit aujourd'hui, ou la «crise» des divers métiers hérités de la tradition. L'analyse typologique aide à formuler cette relation entre les échanges interindividuels, les conduites quotidiennes, le sens donné aux expériences et aux épreuves particulières et les caractéristiques globales des sociétés démocratiques. L'universalité de la citoyenneté est évidemment très abstraite, mais elle a des effets sur les relations les plus personnelles. Le sens des relations entre les individus, le sens que les individus donnent à leurs expériences ne sont pas séparables du projet collectif de la société démocratique. Ce sont ces relations que les typologies aident à percevoir et à formuler.

Dans ce grand et beau livre qu' est pour moi «La relation à l'autre», vous critiquez, avec des arguments d'une étincelante perspicacité, le relativisme absolu, les communautarismes et le multiculturalisme, mais acceptez le relativité des cultures, conçues cependant comme étant à leur tour relatives. Vous revenez sur cette question dans "Qu' est-ce que la citoyenneté?» (2000) et dans les chroniques, si jouteuses, pugnaces, passionnantes, réunies dans «Au fur et à mesure» (2003).

$J$ 'aimerais mieux comprendre comment peut-on accorder la vision universaliste, la nécessité d'un monde commun, les valeurs républicaines, avec les particularismes religieux, historiques, voire avec la reconnaissance de certains droits? Pouvez-vous dire comment y parvenir? 
Le relativisme culturel est un fait, on peut juger qu'il s'agit là d'une richesse ou d'une vertu au nom des valeurs démocratiques, mais peu importe, c'est un fait. On observe d'ailleurs que dans la «mondialisation» actuelle qui entraîne une certaine uniformité au moins des comportements économiques, les systèmes d'interprétation culturelle ne connaissent pas la même tendance à l'homogénéisation, soit que les traditions se maintiennent ou se renouvellent, soit que des nouvelles réactions d'affirmation de soi conduisent à des inventions des groupes pour revendiquer leur identité. Il faut donc prendre le relativisme culturel comme une donnée. La question que mon ami Selim Abou a formulée dans des termes qui m'ont paru lumineux dans son chef d'œuvre, Cultures et droits de l' homme, c'est de savoir si la relativité des cultures est elle-même relative ou absolu, si elle peut interdire toute communication entre les hommes - c'est le relativisme culturel absolu -, ou bien si, par delà les différentiations, il existe un horizon commun à tous les hommes, qui autorise la communication malgré les différences et, en conséquence, justifie que des jugements partiels soient portés sur tel ou tel trait culturel - c'est le relativisme culturel relatif. J'adhère totalement à cette seconde position. Je suis profondément universaliste, c'est-à-dire convaincue qu'il existe bien un horizon d'universalité permettant la communication entre tous les hommes, quelles que soient leur «culture» ou leurs fidélités, à partir de ce qui les caractérise en propre, c'est-à-dire leur liberté. Grâce aux exemples extrêmes de l'excision des petites filles noires et de la traite des Noirs, j'ai réussi à en convaincre certains de mes étudiants, bien qu'avec peine. La fonction des institutions démocratiques est précisément de construire et de faire vivre ces lieux, dans lesquels et par lesquels se rencontrent les hommes, quelle que soit par ailleurs leur fidélité à des traditions particulières. C'est le sens proprement philosophique de la citoyenneté: construire le lieu, au sens concret et au sens abstrait du terme, où peuvent communiquer, au nom des valeurs communes - la liberté et l'égalité des être humains rationnels - ceux qui ont des histoires différentes auxquelles ils sont légitimement attachés. Cela n'est évidemment pas facile, puisque les institutions républicaines ne sont jamais purement républicaines, que l'aspiration universaliste tend toujours à se réifier en religion civile, que les hommes démocratiques ne sont pas toujours fidèles aux valeurs dont ils se réclament et qu'ils sont toujours tentés de devenir plus «ethniques» que «civiques». Mais la citoyenneté est la seule idée dont nous disposions pour donner aux individus le droit de rester fidèles à des traditions particulières et d'organiser concrètement les moyens pour qu'ils puissent entrer en relation avec les autres sur le fondement de l'égale dignité de tous les hommes. C'est la seule idée dont nous disposions pour construire un espace commun, abstrait, dont l'abstraction précisément autorise la vie en commun.

Les menaces sur cette organisation politique sont nombreuses. Respecter les pratiques démocratiques réclame une éducation, donc du temps. Or, les démocraties modernes sont exigeantes et vivent dans l'immédiat. L'adhésion non réfléchie au groupe immédiat et le rejet de l'autre sont plus spontanés, apprendre à surmonter ces sentiments naturels est une condition de la pratique démocratique. D'où mon inquiétude devant ce qu'on appelle les communautarismes. Lorsque l'identification au groupe particulier prime la volonté de rencontrer l'autre dans l'espace commun de la citoyenneté, c'est le principe même de l'intégration des sociétés modernes démocratiques qui est mis en cause. Or c'est une tentation permanente quand s'affaiblit la confiance dans les institutions de la citoyenneté commune. 
Les sociétés démocratiques modernes, dans leur recherche prioritaire du bienêtre des individus, ont tendance à privilégier le particulier ou le «naturel» et le «spontané» au dépens de toute forme de dépassement de ce «naturel» et ce «spontané », c'est-à-dire du civisme. La « démocratie providentielle» risque d'affaiblir le principe civique et de favoriser l'expression des sentiments immédiats au nom de l'authenticité de chacun. J'ai essayé de le montrer dans le livre qui s'efforce d'élaborer le type-idéal de La Démocratie providentielle. Il formule mon inquiétude sur l'évolution des sociétés actuelles. Je les vois menacées par la recherche du confort de l'individu et oublieuses de la nécessité d'affirmer les valeurs de l'espace civique, par définition commun à tous, où les fidélités particulières sont surmontées et dépassées au nom de valeurs proprement politiques. La voie est difficile qui permet de reconnaître les droits de chacun à garder ses convictions particulières et la nécessité de respecter l'espace commun qui permet de «vivre ensemble», pour reprendre l'expression à la mode. Le danger actuel vient de la dissolution de l'espace commun et de l'affaiblissement du politique plus que de la tyrannie du «jacobinisme» ou du «républicanisme» excessif. Le combat de certains «multiculturalistes » ou « régionalistes » contre les institutions nationales vise la situation du passé.

Dans «La Démocratie providentielle. Essai sur l'égalité contemporaine» (2002), vous mettez en évidence l'impact que le déploiement du principe d'égalité a sur la nature, les formes, le sens du lien social et même sur la légitimité politique. Si les citoyens n'arrivent plus à s'identifier à leur Etat-Nation, s'ils n'ont plus un sens véritable du bien commun primant sur leurs intérêts individuels, que peut-on faire pour avoir des valeurs partagées, pour recréer une société où l'intégration politique soit effectivement consistante et opérante? Pour le dire avec vos propres mots : "Comment peut-on continuer à 'faire société'?»

C'est en effet l'interrogation que suscite l'évolution démocratique aujourd'hui. La revendication de l'égalité «réelle», qui implique une intervention croissante de l'Etat pour compenser les effets immédiats du marché, est inscrite dans les valeurs et les aspirations démocratiques d'une société globalement riche. En tant que démocrates, nous la jugeons légitime, nous en faisons même de plus en plus la source principale de la légitimité politique: la société doit répondre aux besoins de ses membres. Mais cette évolution peut avoir pour effet pervers de donner comme seul objectif à la vie commune la satisfaction matérielle et immédiate des besoins des individus. Encore une fois, il ne s'agit pas de critiquer cette vocation de la démocratie de répondre aux besoins de bien-être et d'égalité réelle: c'est la conséquence naturelle de la primauté donnée à l'individu dans les sociétés démocratiques. Mais comme les besoins ne comportent en eux-mêmes pas de limites, qu'ils se renouvellent au fur et à mesure qu'ils sont satisfaits, la démocratie est entraînée dans une dynamique sans fin. Or, c'est en partageant des valeurs politiques communes que les individus peuvent dépasser leurs identifications à des collectivités historiques particulières et leurs intérêts personnels et immédiats. Leurs intérêts économiques opposent naturellement les individus et les groupes; surmonter ces rivalités naturelles implique de partager un projet commun, celui que la citoyenneté nationale a incarné jusqu'à présent. Avec l'affaiblissement de la nation - non remplacée encore par l'Europe - et, en même temps, de l'idée 
civique, le risque est grand de voir dépérir l'unité et la volonté politiques des sociétés démocratiques. Pour compenser, il faudrait que le monde politique retrouve une vertu et une volonté que les conditions de la démocratie ne favorisent pas. D'une certaine façon, je retrouve les thèmes de Tocqueville dans la seconde Démocratie en Amérique, ce livre d'un visionnaire rationnel pour lequel j'ai une admiration sans borne. Il me donne parfois le sentiment que je ne fais que paraphraser ses intuitions fondées, encore une fois, sur une analyse parfaitement rationnelle.

Ici et là vous écrivez d'être perçue quelque peu marginale par la communauté sociologique. Et pourtant vous avez présidé la Société française de sociologie, joué un rôle notable dans la vie de la profession. Vos travaux ont obtenu de nombreuses reconnaissances internationales. Pouvez-vous en dire davantage sur se sentiment de marginalité?

Je ne revendique pas cette marginalité, je la constate. Elle n'a jamais été le produit d'une volonté ou d'une élégance, elle tient sans doute à mon caractère et à une position paradoxale dans le monde de la sociologie, dans lequel les sujets auxquels je me suis attachée sont plus souvent traités par des sociologues «de gauche» et où la dimension politique est marginalisée. En ce qui concerne la Société française de sociologie, elle était elle-même, quand je l'ai présidée entre 1995 et 1999, très marginalisée. Elle était la survivance d'une conception élitiste des sociétés savantes. Depuis sa fondation au début des années 1960, elle avait été présidée par tous les sociologues connus, d'Aron à sa fondation, à Crozier ou Touraine qui la présidait en 1968. Les sociologues français ont toujours été divisés, les rapports entre les différentes «écoles » étaient inexistants ou conflictuels et la Société française de sociologie n'y pouvait rien, elle était dépourvue de tous moyens et de tout pouvoir direct ou même indirect. Pendant ma présidence, j'avais souhaité travailler à ouvrir les sociologues français sur le monde étranger, à faire faire des traductions d'auteurs français, mais le résultat de mes efforts a été nul. Ce titre de présidente d'une société marginalisée confirme l'analyse de ma marginalité.

J'ai été son avant-dernière présidente, avant qu'elle ne meure, deux ans après, de sa belle mort et soit remplacée par l'association actuelle, l'AFS, beaucoup plus nombreuse, beaucoup plus professionnelle, adaptée à ce qu'est devenue la sociologie, une discipline qui doit former des sociologues de métier. La nouvelle association apporte beaucoup d'informations utiles et témoigne d'une activité de réunions et de colloques qui révèle l'évolution de la discipline.

Par ailleurs, je ne crois pas avoir beaucoup contribué à la vie de la profession. J'ai toujours utilisé mon droit de vote à l'EHESS et, à ce titre, j'ai participé à l'élection de certains de mes collègues, parfois avec beaucoup de conviction (je ne citerai pas de noms), parfois avec résignation (j'en citerai encore moins). D'autre part, comme l'immense majorité des sociologues est à gauche, à deux reprises la droite au pouvoir, qui n'avait guère le choix, m'a nommée membre de la Commission du CNRS. La première fois, c'était à la fin des années 1970, la Commission était un lieu d'affrontements violents, les jugements étaient presque exclusivement politiques. D'ailleurs les syndicalistes en faisaient la théorie: «On est aussi capables que vous de porter des jugements scientifiques, mais ce n'est pas 
notre rôle!». On participait pourtant à une commission d'évaluation scientifique. De plus, on présidait à la titularisation des chercheurs «hors statut», ce qui aboutissait à recruter des chercheurs dont certains étaient fort médiocres et incompétents, pendant que d'autres, méritants, ne pouvaient entrer au CNRS. C'était insupportable pour quelqu'un qui, comme moi, crois à la qualité et au travail scientifiques. J'ai eu d'ailleurs fort mauvaise réputation lorsque j'ai demandé à l'un de ces «hors-statut» titularisé qui venait de m'expliquer qu'on ne pouvait traiter de l'identité juive que de manière littéraire pourquoi, dans ce cas, il restait chercheur en sociologie, cela a paru une réflexion «fasciste»! Le comble a été atteint quand la Commission a récupéré un chercheur «révisionniste» exclu à cause de ce révisionnisme de la Commission d'histoire, et travaillant sur les camps soviétiques. Les communistes le soutenaient, car l'application du «révisionnisme» aux camps soviétiques leur paraissait une bonne idée, les «syndicalistes » défendaient toute personne déjà entrée au CNRS sans vouloir admettre qu'un chercheur indigne du CNRS prenait par définition la place d'un chercheur respectable. L'un de mes collègues, particulièrement fin et informé, a ajouté que le candidat ne pouvait être révisionniste, puisqu'il était juif. C'en était trop, j'ai démissionné. Il faudra faire l'histoire du dévoiement d'une Commission dite scientifique du CNRS au cours des années 1970.

La seconde expérience, dix ans après, a été moins malheureuse. Les conflits politiques s'étaient apaisés, la Commission comprenait des chercheurs de grande qualité comme Jean Baechler et Raymond Boudon, j'étais sans doute plus respectée à cause de la publication de plusieurs livres pendant les dix ans qui avaient précédé. J'ai pu ainsi participer au recrutement au CNRS de Serge Paugam et de Sylvie Mesure ainsi qu'à la promotion de Danièle Hervieu-Léger ou de Pierre-Michel Menger. Cela m'a fait plaisir, mais j'ose espérer qu'étant donné leur qualité ils auraient connu le même destin académique sans ma présence.

Je crois que la situation actuelle n'est pas comparable avec ce que j'ai connu. Bien entendu, les Commissions du CNRS sont des lieux de marchandages et de stratégies personnelles ou collectives, c'est inévitable. Mais les candidats à l'entrée sont bien formés, plus professionnels et la dimension idéologique s'est atténuée. L'avenir de la discipline réside dans des travaux sérieux qui développent la connaissance objective de la société. On ne peut attendre d'une discipline académique qu'elle compte des théoriciens comparables aux fondateurs de la pensée sociologique, mais elle doit rassembler des chercheurs sérieux qui produisent des connaissances utiles. Il me semble qu'elle évolue aujourd'hui dans cette direction. Mais il reste en France un handicap sérieux qui subsistera même après le départ à la retraite des anciens « hors statut», c'est la bureaucratisation de la recherche. Les meilleurs chercheurs consacrent un temps disproportionné à répondre aux exigences des diverses bureaucraties, en sorte que le moyen devient la fin.

Pour revenir à ma marginalité, mes travaux ont été peu entendus et peu discutés. La Revue française de sociologie, dont la politique de comptes rendus ne m'est pas claire, ne s'en est pas guère préoccupée. La politisation du milieu, d'un côté, la volonté de rigueur statistique, de l'autre, y ont contribué, mais aussi le fait que les sociologues admettent mal la prise en compte du politique. Outre la sociologie du milieu - le partage du domaine entre les différentes «écoles »-, outre mon caractère personnel et sans doute ma timidité, c'est la raison de fond. Il faut ajouter qu'il existe peu de véritable discussion scientifique dans le milieu 
sociologique. Les différentes «écoles » ont leur domaine et leur lieu de puissance et, entre leurs affidés, c'est plutôt l'ignorance réciproque et le silence méprisant qui sont la règle. Cela contribue à ce que les sociologues soient absents de certains des grands débats menés par les philosophes politiques, ce qui me paraît regrettable, car ils pourraient apporter quelque chose de spécifique. C'est en tous cas ce que j'essaie de faire.

Quant à mes relations internationales, elles ont été également fort limitées. Ma vie de famille et mon caractère - je déteste voyager - m'ont écartée de la fréquentation des colloques et des invitations dans les universités étrangères. J'ai lu de près certains collègues étrangers, comme les sociologues de l'ethnicité aux Etats-Unis et en Grande-Bretagne, Daniel Bell. J'ai beaucoup d'admiration pour Selim Abou et pour Shmuel Eisenstadt, j'ai collaboré à un moment donné avec Pierre Centlivres sur un sujet précis, la nationalité. Mais tout cela a été le fruit du hasard et des rencontres, sans aucune stratégie. De toutes façons, je pense que les véritables échanges passent par une lecture attentive des auteurs et non par les conversations et invitations réciproques. Cette conviction a aussi contribué à limiter ma vie sociale internationale.

Vous avez souvent déploré le peu d'audience que les sociologues ont auprès des classes gouvernantes. Cependant vos recherches ont influencé passablement les travaux de la Commission de la nationalité et c' est bien le sociologue qui a été amené à faire partie du Conseil constitutionnel. Est-ce un hasard, une exception, ou c' est la voie souterraine empruntée par les travaux sociologiques pour donner aux «choix» une certaine rationalité? Comment arrivez-vous à discipliner les inévitables contradictions ou tensions entre l'analyse des réalités sociales et les contraintes normatives du système juridique?

Je garde, comme je vous l'ai dit, un grand souvenir de la Commission de la nationalité, qui a donné un véritable élan à ma réflexion. Il y avait aussi sans doute le sentiment d'être «utile», d'avoir un peu d'influence. Pour un pur universitaire, dépourvu même de tout pouvoir académique, c'est sans doute une occasion qui paraît unique. En plus, c'est un sujet sur lequel j'avais des convictions profondes. Cela dit, bien sûr, d'autres membres de la Commission ont eu au moins autant d'influence que moi. C'est indirectement à la Commission de la Nationalité que je dois d'avoir été nommée au Conseil constitutionnel. Ma nomination est le produit improbable d'une série de circonstances, que j'ai reconstituée mais qui ne mérite pas d'être racontée ici, mais, entre autres éléments, ma participation à la Commission avait convaincu Marceau Long, président de la Commission, et Pierre Mazeaud, futur président du Conseil constitutionnel, que j'étais une sociologue «comestible» avec laquelle on pouvait discuter. Pierre Mazeaud, en même temps que Simone Veil, a donc appuyé ma candidature. Mais elle n'aurait pas été envisagée si Alain Lancelot, par son talent, n'avait pas convaincu les membres du Conseil qu'un spécialiste des sciences humaines pouvait avoir son utilité dans l'élaboration collective des décisions du Conseil. Ce qui prouve que si les sociologues ne se contentent pas du confort de la critique radicale, ils peuvent être quelque peu écoutés. Cela dit, mes collègues sont tout à fait courtois ou même amicaux, mais je me demande s'ils me trouvent utile en tant que sociologue. Il faudrait leur demander. Je n'ai pas le sentiment de vivre de véritables tensions 
entre mon expérience juridictionnelle et l'analyse sociologique. Elles ne sont vraiment pas sur le même plan. Mon collègue Colliard trouve même que j'ai trop de respect pour le droit, mais il me semble que nous sommes là pour appliquer le droit. Dans quelques décisions importantes (les quotas, la «discriminations positives» par exemple), mon choix repose sur mes réflexions sociologiques, alors que les collègues qui sont d'accord avec moi le font pour des raisons plus politiques ou même sentimentales. Mais la frontière entre les deux est fragile. Contribuer à une décision, même si elle n'est pas essentielle (il ne faut pas surestimer le pouvoir du Conseil constitutionnel), continue à me fasciner. D'autre part, j'apprends beaucoup sur les pratiques quotidiennes du monde politique. Ce sera difficile d'utiliser cette expérience, effectivement unique pour un sociologue. Le devoir de réserve m'impose la discrétion même à la fin de mon mandat, l'anonymat est impossible à respecter dans une aussi petite institution. J'essaierai toutefois de ne pas laisser cette expérience en complète jachère sociologique et mes enfants trouveront le journal ethnologique que j'écris régulièrement. Peut-être seront-ils les seuls intéressés par mes récits. 